\title{
Iron-induced luminescence as a method for assessing lipid peroxidation of frozen-thawed goat spermatozoa
}

\author{
P. Gogol ${ }^{\dagger}$, A. Wierzchoś-Hilczer and M. Cegła \\ Department of Biotechnology of Animal Reproduction, National Research Institute of Animal Production, Krakowska 1, 32-083 Balice, Poland
}

(Received 18 September 2006; Accepted 19 March 2007)

\begin{abstract}
Freezing/thawing procedures induce enhanced reactive oxygen species (ROS) formation in mammalian sperm and these ROS may be a cause for the decrease in sperm function following cryopreservation. In the present study, we used a chemiluminescence method to detect ROS-induced damage in goat spermatozoa. Iron-induced luminescence of fresh and frozen/thawed sperm cells was assessed using a luminometer. It was shown that the freezing/thawing procedure had a significant effect on some luminescence parameters. Semen freezing significantly increased the values of integral, peak max, T.half (rise) and T.max (peak) parameters. A significant correlation was observed between the percentage of motile spermatozoa and integral, peak max and T.half (rise) parameters. In conclusion, the results of the present study indicate that measurement of induced luminescence can be an alternative, sensitive and relatively simple method for assessing the effect of cryopreservation on oxidative damage to spermatozoa.
\end{abstract}

Keywords: goats, lipid peroxidation, luminescence, semen, spermatozoa motility

\section{Introduction}

Freezing and thawing, two major steps in cryopreservation of spermatozoa, have a major effect on cell structure and function (Hammerstedt et al., 1990). The freezing-thawing cycle causes damage to the plasma membrane (Hammerstedt et al., 1990), reduces motility and the fertilising ability of spermatozoa (Hammerstedt, 1993) and induces premature capacitation and nuclear decondensation (Cormier et al., 1997). Even in the presence of cryoprotectants such as glycerol, egg yolk and milk, significant structural alterations take place. In recent years, antioxidants have been tested in combination with basic common cryoprotectants to minimise the damage caused by freezing and thawing. The beneficial effects of antioxidants provide indirect evidence that an oxidative stress occurs during cryopreservation (Alvarez and Storey, 1992; Chen et al., 1993; Sanchez-Partida et al., 1997). This has been confirmed by studies which showed that reactive oxygen species (ROS) are produced during freezing and thawing of bovine (Chatterjee and Gagnon, 2001) and equine spermatozoa (Ball et al., 2001).

One of the major biological processes associated with ROS is lipid peroxidation. Lipid peroxidation proceeds with

\footnotetext{
${ }^{\dagger}$ E-mail: pgogol@izoo.krakow.pl
}

the extraction of hydrogen and the formation of a number of reactive intermediates that can result in a chain reaction or propagation of peroxidation within the membrane (Aitken et al., 1993a; Storey, 1997). Mammalian sperm cells are particularly sensitive to oxidative damage due to the high level of easily peroxidisable polyunsaturated fatty acids (Jones et al., 1979) and fairly low activity of the enzymatic anti-oxidative system. The attack of free radicals on unsaturated fatty acid-rich lipids of sperm cell membranes leads to an irreversible decrease in membrane fluidity (Borst et al., 2000), alteration in membrane permeability and metabolism (Jones et al., 1979; Ohyashiki et al., 1988; Ohta et al., 1989) and reduced sperm ability to penetrate the egg (Aitken et al., 1993b; Kodama et al., 1996).

In view of the importance of lipid peroxidation in defective sperm function, quantification of this process is of some diagnostic significance. At present, the most widely used assay for lipid peroxidation involves the measurement of malondialdehyde (MA), a small molecular mass degradation product of peroxidative process that can be measured by virtue of its capacity to form adducts with thiobarbituric acid (Aitken et al., 1993a). Although the method is sensitive and can detect the end-point reaction product of lipid peroxidation, it is relatively elaborate and provides only an indirect measure of lipid peroxidation (Pap et al., 2000). Moreover, MA only accounts for around $5 \%$ of 
the products generated during lipid peroxidation (Marshall et al., 1985). Other extremely toxic lipid peroxidation products such as 4-hydroxynonenal, which are known to be present in semen and to have a powerful inhibitory effect on sperm function, are not accounted for in the MA assay (Selley et al., 1991).

Recently, the application of a fluorescent fatty acid probe, $\mathrm{C}_{11}$-BODIPY $^{581 / 591}$, has been described in a number of studies as a means to monitor lipid peroxidation in living cells (Borst et al., 2000; Pap et al., 2000; Ball and Vo, 2002). The probe shifts from red to green upon oxidation, and the ratio of red and green fluorescence has been used as a measurement of lipid peroxidation. The dye is relatively non-fluorescent in solution and has been evaluated by a fluorescence microplate reader, by epifluorescence microscopy, by confocal microscopy and by flow cytometry (Pap et al., 2000). The fluorescence changes in $\mathrm{C}_{11}$-BODIPY ${ }^{581 / 591}$ reflect indirectly the oxidation of unsaturated fatty acids (Borst et al., 2000).

Chemiluminescence is considered to be an alternative and potentially sensitive method to assess the oxidation or auto-oxidation of lipids (Miyazawa et al., 1994; Albertini and Abuja, 1998). In several studies, the luminescence signal has been correlated with other indicators of lipid peroxidation, such as the MA concentration (Doi et al., 2002), the concentration of exogenously added lipid hydroperoxides (Guajardo et al., 2002) and the content of conjugated dienes (Albertini and Abuja, 1998), confirming that low-level chemiluminescence can serve as an indicator of lipid peroxidative damage. In the case of spermatozoa, however, the intensity of spontaneous luminescence is extremely weak and thus difficult to measure. Earlier studies (Laszczka et al., 1995; Sławiński et al., 1998; Gogol, 2005; Gogol and Szczęśniak-Fabiańczyk, 2006) have shown that recording the iron ion-induced luminescence can be an alternative and relatively simple method of measuring sperm lipid peroxidation.

The aim of the present study was to evaluate the effect of the freezing/thawing process on iron ion-induced luminescence of buck sperm as an indicator of cell oxidative damage.

\section{Material and methods}

\section{Semen}

Five healthy, adult goat bucks were used in this study. Semen was collected from February to May using an artificial vagina. Semen was processed using a modified Corteel cryopreservation procedure (Kareta and Cegła, 1999) in a milk-glycerol extender, frozen in $0.25 \mathrm{ml}$ plastic straws using liquid nitrogen vapour and stored in liquid nitrogen.

Frozen straws were thawed in a $37^{\circ} \mathrm{C}$ water bath for $30 \mathrm{~s}$ immediately before use. Freshly ejaculated semen as well as thawed semen samples from the same bucks were also evaluated for luminescence parameters and sperm progressive motility.

\section{Luminescence measurements}

Luminescence was measured at $20^{\circ} \mathrm{C}$ using a Berthold AutoLumat LB953 luminometer equipped with a cooled photomultiplier with a spectral response range from 370 to $620 \mathrm{~nm}$. Prior to measurement of luminescence, spermatozoa were separated from the seminal plasma and diluents by two-fold centrifugation $(700 \times \mathbf{g}$ for $15 \mathrm{~min})$ and resuspended in $0.9 \% \mathrm{NaCl}$ to a final concentration of $200 \times 10^{6}$ cells per $\mathrm{ml}$.

To $500 \mu \mathrm{l}$ of the washed sperm suspension at a concentration of $200 \times 10^{6}$ cells per ml, $10 \mu \mathrm{l}$ of $5 \mathrm{mmol} / \mathrm{l}$ luminol was added. Emission was induced by adding (using automated injector system) $100 \mu \mathrm{l} 0.3 \mathrm{mmol} / \mathrm{l} \mathrm{FeSO} \mathrm{C}_{4}$ solution (final concentration $0.05 \mathrm{mmol} / \mathrm{l}$ ).

Immediately after injection of $\mathrm{FeSO}_{4}$ solution, light emission kinetics was measured for $300 \mathrm{~s}$ (Figure 1). After complete measurements, the following luminescence parameters were calculated: integral - total integral of the measurement signals (counts $\times 10^{5}$ per integration time); peak $\max \left(\right.$ c.p.s. $\left.\times 10^{3}\right)$ - height of highest peak; slope $\max$ (c.p.s. $\times 10^{2}$ ) - maximum slope value of curve; T.slope $\max$ - time at maximum slope; T.half (rise) - time at half 'peak max' height in ascending direction; T.max (peak) - time at peak maximum; T.half (fall) - time at half 'peak max' height in descending direction.

\section{Assessment of sperm motility}

The percentage of progressive motile spermatozoa was evaluated under a contrast phase microscope equipped with a heated plate at $37^{\circ} \mathrm{C}$.

\section{Statistical analysis}

Results are expressed as means \pm s.e. Data were subjected to variance analysis according to the GLM procedure of the Statistical Analysis Systems Institute (2001).

The significance of differences between means was tested by the least squares method using the LS means procedure. The correlations between luminescence parameters and motility were calculated using Spearman's rank method.

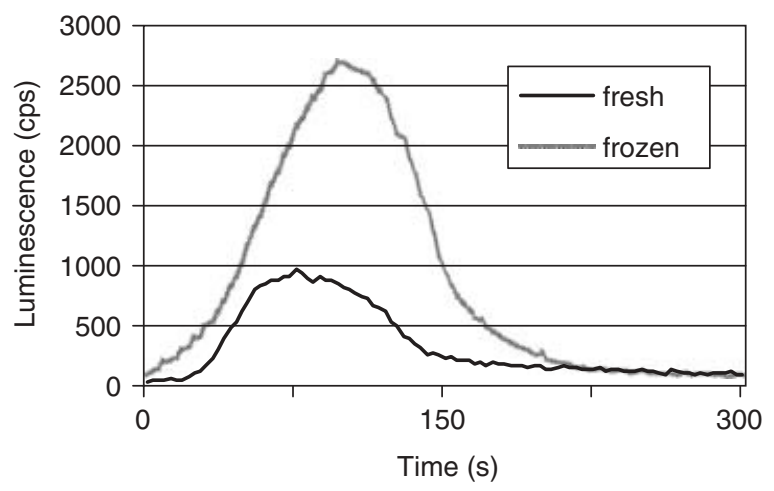

Figure 1 Kinetics of induced luminescence of goat spermatozoa. 


\section{Results}

The effect of freezing/thawing on luminescence parameters and sperm motility is shown in Table 1 . Semen freezing significantly increased the values of integral $(P<0.01)$, peak max $(P<0.01)$, T.half (rise) $(P<0.05)$ and T.max (peak) $(P<0.05)$ parameters. No significant differences between fresh and frozen sperm in the values of slope max, T.slope max and T.half (fall) parameters were observed.

Table 1 The effect of freezing-thawing on luminescence parameters and motility of goat spermatozoa (mean \pm s.e.)

\begin{tabular}{lcr}
\hline \hline & \multicolumn{2}{c}{ Semen } \\
\cline { 2 - 3 } Parameter & \multicolumn{2}{c}{ Fresh } \\
& $1.90^{\mathrm{A}} \pm 0.16$ & \multicolumn{1}{c}{ Frozen } \\
\hline Integral & $2.03^{\mathrm{A}} \pm 0.30$ & $6.43^{\mathrm{B}} \pm 0.96$ \\
Peak max & $1.46 \pm 0.33$ & $8.86^{\mathrm{B}} \pm 1.76$ \\
Slope max & $5.96 \pm 0.43$ & $8.27 \pm 4.14$ \\
T.slope max & $35.71^{\mathrm{a}} \pm 3.51$ & $10.32 \pm 2.20$ \\
T.half (rise) & $69.14^{\mathrm{a}} \pm 4.67$ & $54.29^{\mathrm{b}} \pm 6.61$ \\
T.max (peak) & $131.40 \pm 13.03$ & $92.00^{\mathrm{b}} \pm 7.80$ \\
T.half (fall) & $82.3^{\mathrm{A}} \pm 3.08$ & $141.71 \pm 7.74$ \\
Motility (\%) & $51.7^{\mathrm{B}} \pm 4.57$ \\
\hline \hline
\end{tabular}

$\overline{a, b}$ Means within a row with different superscripts are significantly different at $P<0.05$.

$A, B$ Means within a row with different superscripts are significantly different at $P<0.01$.

Table 2 Correlations between luminescence parameters and sperm motility

\begin{tabular}{lc}
\hline \hline Parameter & Motility $^{+}$ \\
\hline Integral & $-0.79849(P<0.0001)$ \\
Peak max & $-0.74061(P<0.0001)$ \\
Slope max & $-0.25192(P=0.1792)$ \\
T.slope max & $-0.14537(P=0.4434)$ \\
T.half (rise) & $-0.44171(P=0.0145)$ \\
T.max (peak) & $-0.31138(P=0.0939)$ \\
T.half (fall) & $-0.04666(P=0.8101)$ \\
\hline \hline
\end{tabular}

${ }^{+} P<0.05$ was considered significant.
The proportion of motile spermatozoa decreased after semen freezing by $30.6 \%(P<0.01)$.

A significant correlation was observed between the percentage of motile spermatozoa and integral, peak max and T.half (rise) parameters (Table 2). Integral $(r=-0.80)$ and peak $\max (r=-0.74)$ were luminescence parameters that were the most strongly correlated with sperm motility. No significant correlation was found between T.max (peak) and T.half (fall) parameters and the percentage of motile spermatozoa.

Differences between males in the luminescence parameters were observed (Tables 3 and 4). In both fresh and frozen semen, the sperm of buck $C$ was characterised by the lowest, and the sperm of buck B by the highest, values of integral and peak max parameters.

\section{Discussion}

In the present study, induced luminescence (photon emission) measurements were used to determine oxidative damage to goat spermatozoa. Earlier studies covering measurements of the spectral distribution of sperm emission and analysis of the relationships between the concentration of Fe ions and intensity of induced luminescence show that this biophysical phenomenon is strictly related to lipid peroxidation (Sławiński et al., 1998; Gogol, 2005). Ferrous ions have been used extensively to induce rapid lipid peroxidation in a variety of cell types including spermatozoa (Jones et al., 1979; Aitken et al., 1993a; Storey, 1997; Gomez et al., 1998). The ferrous ion promotes the catalysis of lipid peroxides to alkoxyl and peroxyl radicals, which appear to be important in the propagation of the chain reaction of lipid peroxidation in the sperm membrane (Aitken et al., 1993a). In these radical chain reactions, electron-excited molecules are generated and then radiatively deactivated, which manifests itself as an emission of light (chemiluminescence, ultraweak photon emission). Our study is evidence that the kinetics and intensity of induced lipid peroxidation of sperm can be observed based on changes in the intensity of luminescence recorded. Our

Table 3 Results of luminescence measurement and sperm motility for fresh semen (mean \pm s.e.)

\begin{tabular}{lccrrr}
\hline \hline & \multicolumn{5}{c}{ Buck (no. of ejaculates) } \\
\cline { 2 - 6 } Parameter & \multicolumn{1}{c}{$\mathrm{A}(6)$} & $\mathrm{B}(2)$ & $\mathrm{C}(5)$ & $\mathrm{D}(6)$ & $\mathrm{E}(2)$ \\
\hline Integral & $2.4^{\mathrm{A}} \pm 0.3$ & $2.7^{\mathrm{aAC}} \pm 0.8$ & $1.3^{\mathrm{bB}} \pm 0.2$ & $1.5^{\mathrm{bcBC}} \pm 0.1$ & $2.4^{\mathrm{aC}} \pm 0.1$ \\
Peak max & $3.1^{\mathrm{aA}} \pm 0.5$ & $3.2^{\mathrm{ab}} \pm 2.2$ & $0.8^{\mathrm{CB}} \pm 0.1$ & $1.6^{\mathrm{bc}} \pm 0.3$ & $2.2 \pm 0.3$ \\
Slope max & $1.0^{\mathrm{a}} \pm 0.3$ & $0.8 \pm 0.1$ & $0.9^{\mathrm{a}} \pm 0.5$ & $2.9^{\mathrm{b}} \pm 0.8$ & $0.9 \pm 0.1$ \\
T.slope max & $6.1 \pm 1.0$ & $4.1 \pm 0.4$ & $6.0 \pm 0.9$ & $5.9 \pm 0.6$ & $7.4 \pm 2.4$ \\
T.half (rise) & $45.5^{\mathrm{A}} \pm 7.1$ & $48.0^{\mathrm{a}} \pm 6.0$ & $40.2^{\mathrm{a}} \pm 3.1$ & $19.5^{\mathrm{bB}} \pm 5.2$ & $31.5 \pm 1.5$ \\
T.max (peak) & $72.5^{\mathrm{a}} \pm 7.9$ & $87.0^{\mathrm{A}} \pm 15.0$ & $86.4^{\mathrm{A}} \pm 5.3$ & $47.0^{\mathrm{bB}} \pm 6.1$ & $64.5 \pm 1.5$ \\
T.half (fall) & $115.5^{\mathrm{aC}} \pm 11.0$ & $186.0^{\mathrm{ab}} \pm 81.0$ & $189.0^{\mathrm{bA}} \pm 37.2$ & $92.5^{\mathrm{CB}} \pm 6.0$ & $126.0 \pm 12.0$ \\
Motility (\%) & $85.0^{\mathrm{aA}} \pm 2.9$ & $57.5^{\mathrm{B}} \pm 2.5$ & $91.7^{\mathrm{AC}} \pm 1.7$ & $88.0^{\mathrm{AC}} \pm 2.0$ & $75.0^{\mathrm{bAD}} \pm 5.0$ \\
\hline \hline
\end{tabular}

$a, b, c$ Means within a row with different superscripts are significantly different at $P<0.05$.

$A, B, C, D$ Means within a row with different superscripts are significantly different at $P<0.01$. 
Table 4 Results of luminescence measurement and sperm motility for frozen semen (mean \pm s.e.)

\begin{tabular}{|c|c|c|c|c|c|}
\hline \multirow[b]{2}{*}{ Parameter } & \multicolumn{5}{|c|}{ Buck (no. of ejaculates) } \\
\hline & $A(6)$ & B (2) & $C(5)$ & $\mathrm{D}(6)$ & $E(2)$ \\
\hline Integral & $7.4 \pm 1.3$ & $12.1^{\mathrm{aA}} \pm 5.5$ & $2.8^{\mathrm{bB}} \pm 0.9$ & $5.5^{b c} \pm 1.3$ & $9.7^{\mathrm{ac}} \pm 4.7$ \\
\hline Peak max & $10.3 \pm 2.3$ & $17.6^{\mathrm{a}} \pm 11.3$ & $3.0^{\mathrm{b}} \pm 1.4$ & $7.2 \pm 2.4$ & $15.6 \pm 10.8$ \\
\hline Slope max & $5.7 \pm 5.0$ & $0.6 \pm 0.1$ & $0.8 \pm 0.2$ & $22.0 \pm 12.7$ & $1.2 \pm 0.5$ \\
\hline T.slope max & $12.2 \pm 5.6$ & $5.7 \pm 2.1$ & $6.7 \pm 0.8$ & $13.2 \pm 5.4$ & $9.7 \pm 1.5$ \\
\hline T.half (rise) & $44.0^{\mathrm{A}} \pm 4.8$ & $121.5^{B} \pm 7.5$ & $58.8^{\mathrm{A}} \pm 7.6$ & $36.5^{A} \pm 12.5$ & $60.0^{A} \pm 0.0$ \\
\hline T.max (peak) & $77.0^{\mathrm{A}} \pm 6.8$ & $157.5^{\mathrm{aB}} \pm 13.5$ & $110.4^{b} \pm 4.1$ & $70.5^{\mathrm{cA}} \pm 17.4$ & $90.0^{b c} \pm 3.0$ \\
\hline T.half (fall) & $120.0^{\mathrm{aA}} \pm 7.8$ & $196.5^{\mathrm{bB}} \pm 25.5$ & $160.8^{b c} \pm 7.0$ & $131.0^{\mathrm{ac}} \pm 17.0$ & $136.5 \pm 22.5$ \\
\hline Motility (\%) & $55.0 \pm 7.64$ & $35.0^{A} \pm 15.0$ & $75.0^{\mathrm{aB}} \pm 7.64$ & $47.0^{b} \pm 4.64$ & $40.0^{b} \pm 10.00$ \\
\hline
\end{tabular}

$a, b, c$ Means within a row with different superscripts are significantly different at $P<0.05$.

${ }^{\mathrm{A}, \mathrm{B}}$ Means within a row with different superscripts are significantly different at $P<0.01$.

method enables the total level of ROS (generated during the lipid peroxidation process) to be determined (integral parameter) as well as the observation of the reaction kinetics probably related to the antioxidant capacity of sperm. It is supposed that higher sperm antioxidant activity gives flatter kinetic curve. This means the lower peak max and slope max values and the bigger difference between T.half (rise) and T.half (fall). Dissection of the luminescence signal into more parameters then only the integral one makes possible to obtain more detailed information about the processes that take place in the sperm cells.

The rapid increase in luminescence intensity after freezing/thawing of semen, which was accompanied by a decrease in sperm motility, demonstrates that the phospholipids present in goat spermatozoa readily undergo peroxidation.

The decreased motility of spermatozoa may occur due to the action of free radicals under oxidative stress. There are several possible mechanisms behind the decreased motility of spermatozoa connected with oxidative stress. The most often cited is peroxidation of membrane lipids (Aitken et al., 1989, 1993a and 1993b). The attack of free radicals on the unsaturated fatty acid-rich lipids of sperm cell membranes leads to irreversible reduction of membrane fluidity and to the damage of cell membrane-related ATPases, which are responsible for regulation of the intracellular level of ions necessary to maintain normal sperm motility (Ohta et al., 1989).

The lipid peroxidation process in spermatozoa leads to the creation of substances having cytotoxic properties, such as MA and 4-hydroxynonenol (Aitken et al., 1995). Low concentrations of these substances have been shown to inhibit a large number of cellular enzymes and functions, including anaerobic glycolysis limiting ATP generation by the sperm cell (Comporti, 1989).

De Lamiranda and Gagnon (1992) suggest that ROS are responsible for the loss of spermatozoal motility through decreased phosphorylation of axonemal proteins required for sperm movement.

The above free radical processes that occur under oxidative stress conditions can explain the relationships between photon emission parameters and sperm motility.
A similar relationship between the potential for ironinduced MA generation as an indicator of lipid peroxidation and human sperm movement was reported by Kobayashi et al. (1991) and Aitken et al. (1993a).

Analysis of the effect of freezing on the values of particular luminescence parameters and the correlation between sperm motility and luminescence parameters indicate that integral and peak max are the luminescence parameters particularly useful for determining the ROS-induced damage at the level of the sperm plasma membrane.

The large individual differences shown between luminescence parameters (which determine the sperm sensitivity to lipid peroxidation) show the possibility of using luminescence measurements when selecting males whose semen is highly suitable for freezing.

In conclusion, our findings confirm that an oxidative stress occurs during semen cryopreservation and demonstrate that measurement of induced luminescence can be a sensitive and relatively simple method for assessing the effect of freezing and thawing on oxidative damage to spermatozoa.

\section{References}

Aitken RJ, Clarkson JS and Fisher S 1989. Generation of reactive oxygen species, lipid peroxidation and human sperm function. Biology of Reproduction 41, 183-197.

Aitken RJ, Harkiss D and Buckingham DW 1993a. Analysis of lipid peroxidation mechanisms in human spermatozoa. Molecular Reproduction and Development 35, 302-315.

Aitken RJ, Harkiss D and Buckingham DW 1993b. Relationship between ironcatalysed lipid peroxidation potential and human sperm function. Journal of Reproduction and Fertility 98, 257-265.

Aitken RJ, Paterson M, Fisher H, Buckingham DW and Van Duin M 1995. Redox regulation of tyrosine phosphorylation in human spermatozoa and its role in the control of human sperm function. Journal of Cell Science 108, 2017-2025.

Albertini R and Abuja PM 1998. Monitoring of low-density lipoprotein oxidation by low-level chemiluminescence. Free Radical Research 29, 75-83.

Alvarez JG and Storey BT 1992. Evidence of increased lipid peroxidative damage and loss of superoxide dismutase activity as a mode of sublethal cryodamage to human sperm during cryopreservation. Journal of Andrology 13, 232-241.

Ball BA, Vo AT and Baumber J 2001. Generation of reactive oxygen species by equine spermatozoa. American Journal of Veterinary Research 62, 508-515. 
Ball BA and Vo A 2002. Detection of lipid peroxidation in equine spermatozoa based upon the lipophilic fluorescent dye $C_{11}-B_{0 D I P Y}{ }^{51 / 591}$. Journal of Andrology 23, 259-269.

Borst JW, Visser NV, Kouptsova 0 and Visser AJ 2000. Oxidation of unsaturated phospholipids in membrane bilayer mixtures is accompanied by membrane fluidity changes. Biochimica et Biophysica Acta 1487, 61-73.

Chatterjee S and Gagnon C 2001. Production of reactive oxygen species by spermatozoa undergoing cooling, freezing, and thawing. Molecular Reproduction and Development 59, 451-458.

Chen Y, Foote RH and Brockett CC 1993. Effect of sucrose, trehalose, hypotaurine, taurine, and blood serum on survival of frozen bull sperm. Cryobiology 30, 423-431.

Comporti M 1989. Three models of free radical-induced cell injury. ChemicoBiological Interactions 72, 1-56.

Cormier N, Sirard MA and Beiley JL 1997. Premature capacitation of bovine spermatozoa is initiated by cryopreservation. Journal of Andrology 18, 461-468. De Lamiranda E and Gagnon C 1992. Reactive oxygen species and human spermatozoa. II. Depletion of adenosine triphosphate plays an important role in the inhibition of sperm motility. Journal of Andrology 13, 379-386.

Doi H, Iwasaki H, Masubuchi Y, Nishigaki R and Horie T 2002. Chemiluminescence associated with the oxidative metabolism of salicylic acid in rat liver microsomes. Chemico-Biological Interactions 140, 109-119.

Gogol P 2005. Iron-induced luminescence of boar spermatozoa cells. Annals of Animal Science 5, 91-98.

Gogol P and Szczęśniak-Fabiańczyk B 2006. Effect of long-term storage on induced photon emission of boar spermatozoa. Czech Journal of Animal Science 51, 61-65.

Gomez E, Irvine DS and Aitken RJ 1998. Evaluation of a spectrophotometric assay for the measurement of malondialdehyde and 4-hydroxyalkenals in human spermatozoa: relationship with semen quality and sperm function. International Journal of Andrology 21, 81-94.

Guajardo MH, Terrasa AM and Catala A 2002. Retinal fatty acid binding protein reduce lipid peroxidation stimulated by long-chain fatty acid hydroperoxideson rod outer segments. Biochimica et Biophysica Acta 1581, 65-74.

Hammerstedt RH 1993. Maintenance of bioenergetic balance in sperm and prevention of lipid peroxidation: review of the effect on design of storage preservation systems. Reproduction, Fertility and Development 5, 675-690.

Hammerstedt RH, Graham JK and Nolan JP. 1990. Cryopreservation of mammalian sperm: what we ask them to survive? Journal of Andrology 11, 73-88.

Jones R, Mann T and Sherins RJ 1979. Peroxidative breakdown of phospholipids in human spermatozoa, spermicidal properties of fatty acid peroxides, and protective action of seminal plasma. Fertility and Sterility 31 531-537.

Kareta W and Cegła M 1999. Mrożenie nasienia kozłów. Materiały instruktażowe. Instytut Zootechniki, Kraków, Poland.

Kobayashi T, Miyazaki T, Natori M and Nozawa S 1991. Protective role of superoxide dismutase in human sperm motility: superoxide dismutase activity and lipid peroxide in human seminal plasma and spermatozoa. Human Reproduction (Oxford, England) 6, 987-991.

Kodama H, Kuribayashi $Y$ and Gagnon C 1996. Effect of sperm lipid peroxidation on fertilization. Journal of Andrology 17, 151-157.

Laszczka A, Godlewski M, Kwiecińska T, Rajfur Z, Sitko D, SzczęśniakFabiańczyk B and Sławiński J 1995. Ultraweak luminescence of spermatozoa. Current Topics in Biophysics 19, 20-31.

Marshall PJ, Warso MA and Lands WEM 1985. Selective microdetermination of lipid hydroperoxides. Analytical Biochemistry 145, 192-199.

Miyazawa T, Fujimoto K and Usuki R 1994. Rapid estimation of peroxide content of soybean oil by measuring thermoluminescence. Journal of the American Oil Chemists' Society 71, 343-345.

Ohta A, Mohri T and Ohyashiki T 1989. Effect of lipid peroxidation on membrane-bound $\mathrm{Ca}^{2+}$-ATPase activity of the intestinal brush-border membranes. Biochimica et Biophysica Acta 984, 151-157.

Ohyashiki T, Ohtsuka T and Mohri T 1988. Increase of the molecular rigidity of the protein conformation in the intestinal brush-border membranes by lipid peroxidation. Biochimica et Biophysica Acta 939, 383-392.

Pap EH, Drummen GP, Post JA, Rijken PJ and Wirtz KW 2000. Fluorescent fatty acid to monitor reactive oxygen in single cells. Methods in Enzymology 319, 603-612.

Sanchez-Partida LG, Setchell BP and Maxwell WMC 1997. Epididymal compounds and antioxidants in diluents for the frozen storage of ram spermatozoa. Reproduction, Fertility, and Development 9, 689-696.

Selley ML, Lacey MJ, Bartlett MR, Copeland CM and Ardlie NG 1991. Content of significant amounts of a cytotoxic end-product of lipid peroxidation in human semen. Journal of Reproduction and Fertility 92, 291-298.

Sławiński J, Godlewski M, Gumińska M, Kędryna T, Kwiecińska T, Laszczka A, Szczęśniak-Fabiańczyk B and Wierzuchowska D 1998. Stress-induced peroxidation and ultraweak photon emission of spermatozoa cells. Current Topics in Biophysics 22, 195-203.

Statistical Analysis Systems Institute 2001. SAS user's guide statistics, version 8.2. SAS Institute Inc., Cary, NC, USA.

Storey BT 1997. Biochemistry of the induction and prevention of lipoperoxidative damage in human spermatozoa. Molecular Human Reproduction 3, 203-213. 